\title{
Oral Recombinant Methioninase Combined with Caffeine and Doxorubicin Induced Regression of a Doxorubicin-resistant Synovial Sarcoma in a PDOX Mouse Model
}

\author{
TAKASHI HIGUCHI ${ }^{1,2,3}$, KEI KAWAGUCHI ${ }^{1,2}$, KENTARO MIYAKE $^{1,2}$, QINGHONG HAN $^{1}$, \\ YUYING TAN $^{1}$, HIROMICHI OSHIRO ${ }^{1,2}$, NORIHIKO SUGISAWA ${ }^{1,2}$, ZHIYING ZHANG $^{1,2}$, \\ SAHAR RAZMJOOEI ${ }^{1}$, NORIO YAMAMOTO ${ }^{3}$, KATSUHIRO HAYASHI $^{3}$, HIROAKI KIMURA $^{3}$, \\ SHINJI MIWA ${ }^{3}$, KENTARO IGARASHI ${ }^{3}$, SANT P. CHAWLA ${ }^{4}$, ARUN S. SINGH ${ }^{5}$, FREDERICK C. EILBER ${ }^{6}$, \\ SHREE RAM SINGH ${ }^{7}$, HIROYUKI TSUCHIYA ${ }^{3}$ and ROBERT M. HOFFMAN ${ }^{1,2}$ \\ ${ }^{1}$ AntiCancer, Inc., San Diego, CA, U.S.A.; \\ ${ }^{2}$ Department of Surgery, and Divisions of ${ }^{5}$ Hematology-Oncology and \\ ${ }^{6}$ Surgical Oncology, University of California, San Diego, CA, U.S.A.; \\ ${ }^{3}$ Department of Orthopedic Surgery, Kanazawa University, Kanazawa, Japan; \\ ${ }^{4}$ Sarcoma Oncology Center, Santa Monica, CA, U.S.A.; \\ ${ }^{7}$ Basic Research Laboratory, National Cancer Institute, Frederick, MD, U.S.A.
}

\begin{abstract}
Background/Aim: Synovial sarcoma (SS) is a recalcitrant neoplasm with low chemosensitivity. We recently reported that recombinant methioninase (rMETase) inhibited SS growth in a patient-derived orthotopic xenograft (PDOX) mouse model and was more effective when administered in combination with the first-line drug doxorubicin (DOX). Caffeine enhances the efficacy of anticancer drugs by overcoming drug-induced cell-cycle arrest and increasing subsequent apoptosis. Here, we determined the efficacy of oral recombinant methioninase (o-rMETase) in combined with caffeine on an SS-PDOX model. Materials and Methods: Mice bearing SS-PDOX tumors were randomized into four treatment groups of six: Untreated control; o-rMETase alone; o-rMETase with caffeine; DOX plus o-rMETase with caffeine. Tumor size and body weight were measured during the treatment and plasma L-methionine (MET) levels were measured at the end of treatment. Results: All treatments significantly inhibited SS-PDOX tumor growth. Combining
\end{abstract}

Correspondence to: Robert M. Hoffman, AntiCancer, Inc.; 7917 Ostrow St., San Diego, CA 92111, USA. E--mail: all@anticancer.com email: all@anticancer.com; Hiroyuki Tsuchiya, Department of Orthopaedic Surgery, Kanazawa University, 13-1, Takara-machi, Kanazawa 920-8641, Japan. E-mail: tsuchi@med.kanazawa-u.ac.jp; Shree Ram Singh, Basic Research Laboratory, National Cancer Institute, Frederick, MD, 21702, USA. E-mail: singhshr@mail.nih.gov

Key Words: Synovial sarcoma, PDOX, oral rMETase, caffeine, doxorubicin, regression. caffeine with o-rMETase was more effective than o-rMETase alone. DOX combined with o-rMETase and caffeine led to regression of SS-PDOX. Plasma MET levels were reduced with o-rMETase treatment. Conclusion: These results suggest that combining o-rMETase and caffeine along with first-line chemotherapy can be highly effective for SS and has clinical potential for this recalcitrant disease.

Synovial sarcoma (SS) is an aggressive sarcoma subtype that accounts for $5 \%$ to $10 \%$ of all soft-tissue sarcomas (1). Compared with other soft-tissue sarcomas, SS occurs predominantly in younger adults and more than half of patients are teenagers and young adults (2). SS arises almost anywhere in the body and has high tendency to metastasize to the lungs and lymph nodes $(1,2)$. SS is recalcitrant to chemotherapy, including first-line DOX (1-3).

To individualize therapy of patients with cancer, we developed patient-derived orthotopic xenograft (PDOX) mouse models of sarcoma, including SS, as well as other cancer types (4-8). While finding more efficacious agents for SS using PDOX models, we recently found that our developmental therapeutic, rMETase (6-10) can inhibit SS growth in PDOX models when administered orally and increase the efficacy of DOX (7).

Caffeine enhances the efficacy of certain anticancer drugs by overcoming drug-induced cell-cycle arrest and increasing subsequent apoptosis in cancer cells $(11,12)$. Cell-cycle arrest is a survival mechanism in chemotherapy-treated cells (11, 13) and resting cancer cells display enhanced treatment resistance compared with actively cycling cells, as most of 


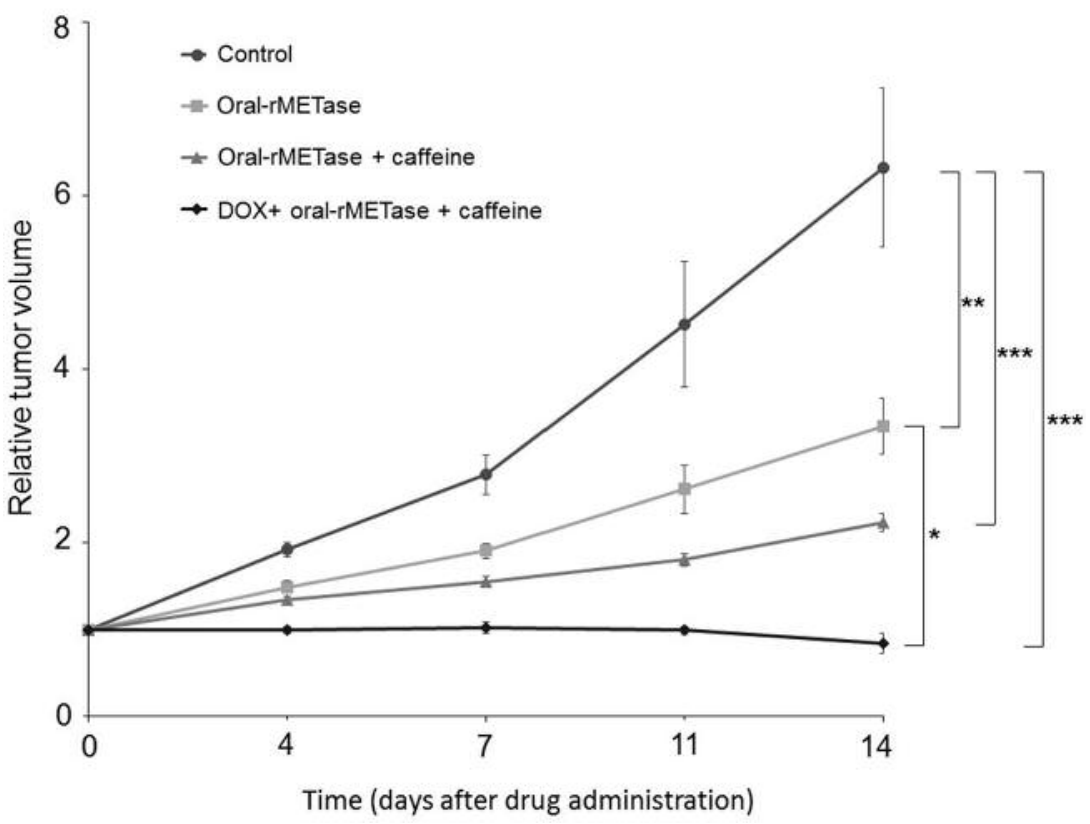

Figure 1. Efficacy of oral recombinant methioninase (o-rMETase) alone, o-rMETase combined with caffeine, and doxorubicin (DOX) combined with o-rMETase and caffeine on a synovial sarcoma patient-derived orthotopic xenograft. Line graphs indicate tumor volume at each time point after the onset of treatment relative to the initial tumor volume for each treatment and control group. $n=6$ mice/group. Significantly different at $* p<0.05$, $* * p<0.01, * * * p<0.001$. Error bars: \pm SEM.

anticancer drugs target the active cell cycle (13). Reacceleration of the cell cycle such as that induced by caffeine can re-sensitize cancer cells to chemotherapy $(11,12)$.

Restriction of methionine (MET) by rMETase arrests cancer cells in $S / G_{2}$ with subsequent apoptosis with or without anticancer drugs (6-10). rMETase targets the elevated requirement of cancer cells for MET, termed MET dependence (9). Caffeine may increase the sensitivity of cancer cells arrested by MET restriction (MR) by inducing mitotic catastrophe (11). In the present study, we investigated the efficacy of combination treatment of o-rMETase and caffeine and determined if this combination is able to overcome DOX resistance in an SS PDox mocel, using our newly developed oral formulation of rMETase $(10,14)$.

\section{Materials and Methods}

Mice. In this study, athymic male $n u / n u$ nude mice (AntiCancer, Inc., San Diego, CA, USA), 4-6 weeks old, were used. Experimental procedures and data collection were carried out as per as our previous publications $(10,14)$. Mouse housing, feeding, surgical processes and imaging were conducted as described elsewhere $(10,14)$. To minimize any suffering of the animals, anesthesia and analgesics were used for all surgical experiments. The mouse investigations presented here were carried out using an AntiCancer, Inc. Institutional Animal Care and Use Committee (IACUC) protocol specifically approved for this study as previously described $(5,10,14)$ and as per as the principles and procedures provided in the National Institute of Health Guide for the Care and Use of Animals under Assurance Number A3873-1 (10, 14).
Patient-derived tumor. Primary SS was obtained from the lower leg of a 45-year-old male (7). The patient underwent surgical resection at the Department of Surgery, University of California, Los Angeles (UCLA) (7). Written informed consent was obtained from the patient as part of a UCLA Institutional Review Board (IRB \#10001857)-approved protocol (7). The patient had received neoadjuvant DOX-based chemotherapy prior to surgery (7).

Surgical orthotopic implantation (SOI) for establishment of the SSPDOX model. All experimental protocols and data were collected as described elsewhere (7). SS sample collection from the patient and transportation to AntiCancer, Inc. were described elsewhere (7). Procedures for making small fragments from tumor samples for subcutaneous transplantation in nude mice have been described (7). Orthotopic implantation to establish the SS-PDOX model has been described (7). The procedure to close wound has been described (7).

rMETase production. Recombinant L-methionine $\alpha$-deamino- $\gamma$ mercapto-methane lyase (rMETase) is a homotetrameric PLP enzyme of 172-kDa molecular mass. The pAC-1 rMETase highexpression clone (AntiCancer, Inc., San Diego, CA, USA) (15), constructed in our laboratory, was used for rMETase production. The fermentation procedure for host recombinant Escherichia coli cells and the purification protocol for rMETase were the same as previously described: rMETase was purified by three different steps using columns including DEAE Sepharose FF (Pharmacia, Uppsala, Sweden), Sephacryl S-200HR (Sigma, St. Louis, MO, USA), and ActiClean Etox (Sigma), which is designed for eliminating endotoxin (15).

Treatment study design in the SS-PDOX model. The SS-PDOX mouse models were randomized into four treatment groups of six 

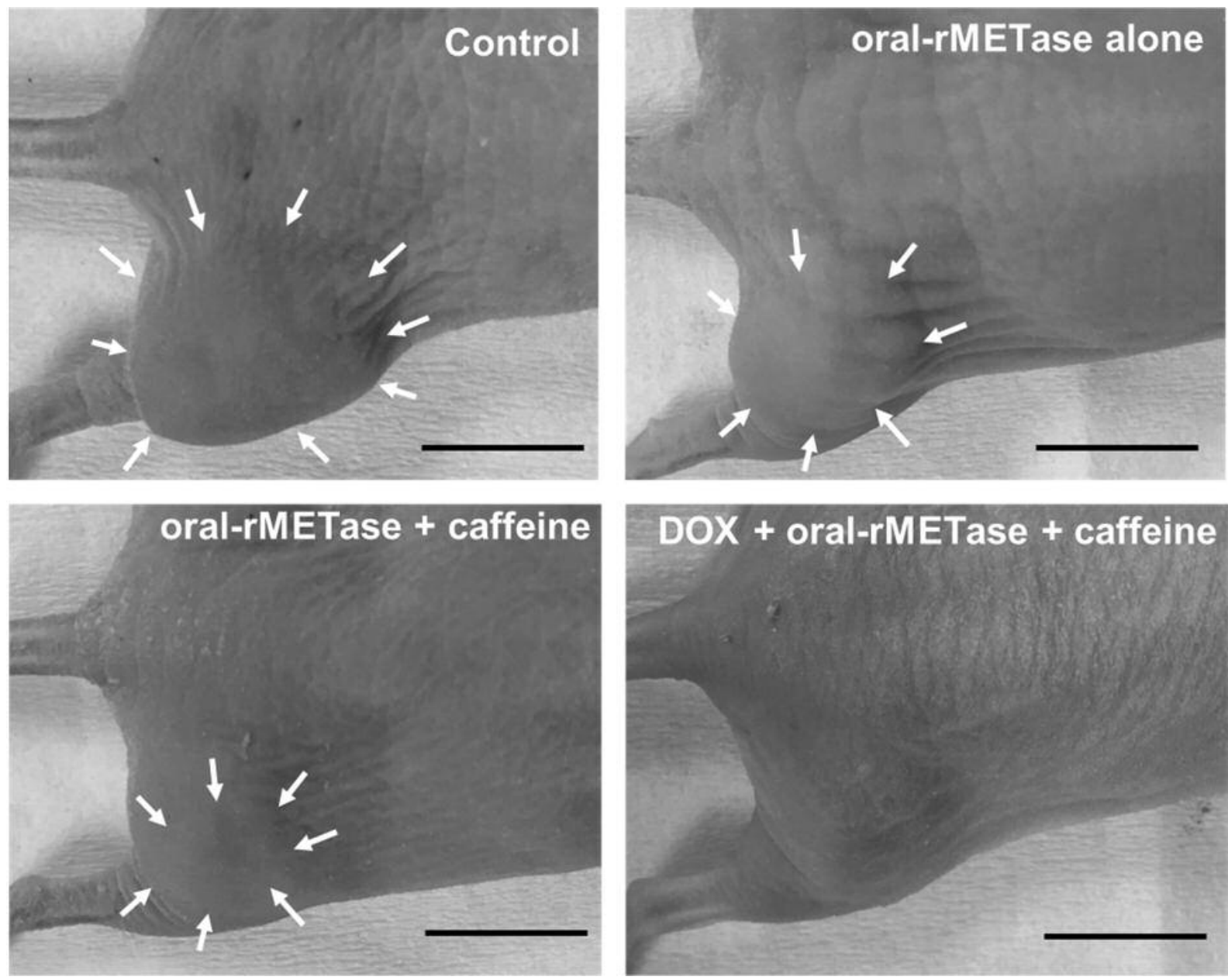

Figure 2. Photographs of representative tumors in patient-derived orthotopic xenograft mouse models from each treatment group at the end of treatment. Arrows indicate the boundary of the tumors. No tumors were detected from the surface in mice treated with doxorubicin (DOX) combined with oral recombinant methioninase (o-rMETase) and caffeine. Scale bars: $10 \mathrm{~mm}$.

mice each: Untreated control without treatment; oral-rMETase (o-rMETASE) alone at 100 units/day for 14 consecutive days; oral o-rMETase combined with caffeine at $100 \mathrm{mg} / \mathrm{kg} /$ day for 14 consecutive days; DOX at $3 \mathrm{mg} / \mathrm{kg}$, intraperitoneal injection, once a week for 2 weeks combined with oral o-rMETase and caffeine. Treatment started when all tumors reached $100 \mathrm{~mm}^{3}$. Tumor length, width and mouse body weight were measured twice per week. Tumor volume was calculated by the following formula: Tumor volume $\left(\mathrm{mm}^{3}\right)=$ length $(\mathrm{mm}) \times$ width $(\mathrm{mm}) \times$ width $(\mathrm{mm}) \times 1 / 2$. Data are presented as the mean \pm standard error of the mean (SEM). The tumor volume inhibition and regression were defined as the tumor volume at each point relative to pre-treatment tumor volume.

Plasma L-methionine (MET) levels. Whole blood was collected from each of three mice in G1 to 3 into heparinized tubes on the final date of treatment (day 14). Plasma was obtained from the blood by centrifugation at 4,000 rpm for 5 minutes. Plasma MET levels were measured with an HPLC (Hitachi L-6200A Intelligent pump; Hitachi, Ltd., Tokyo, Japan) after derivatization of serum amino acids with the fluoraldehyde reagent OPA as described previously (15).

Statistical analysis. All statistical analyses were performed by statistical software EZR (Saitama Medical Center, Jichi Medical University, Saitama, Japan), which is a graphical user interface for $\mathrm{R}$ (The $\mathrm{R}$ Foundation for Statistical Computing, version 3. 4. 1.). It is a modified version of $\mathrm{R}$ commander (version 2. 4-0) including statistical functions for biostatistics. Normal distribution was assessed by the Shapiro-Wilk test. Bartlett's test was used to verify the homogeneity of variances across groups. One-way ANOVA with Tukey HSD for post hoc analysis was used for parametric test of intragroup differences. The Kruskal-Wallis with Steel-Dwass test for post hoc analysis was used for the non-parametric test of intragroup differences. All $p$-values were two-sided and $p$-values of 0.05 or less were considered statistically significant.

\section{Results}

Efficacy of o-rMETase alone, o-rMETase combined with caffeine, and DOX combined with rMETase and caffeine on the 


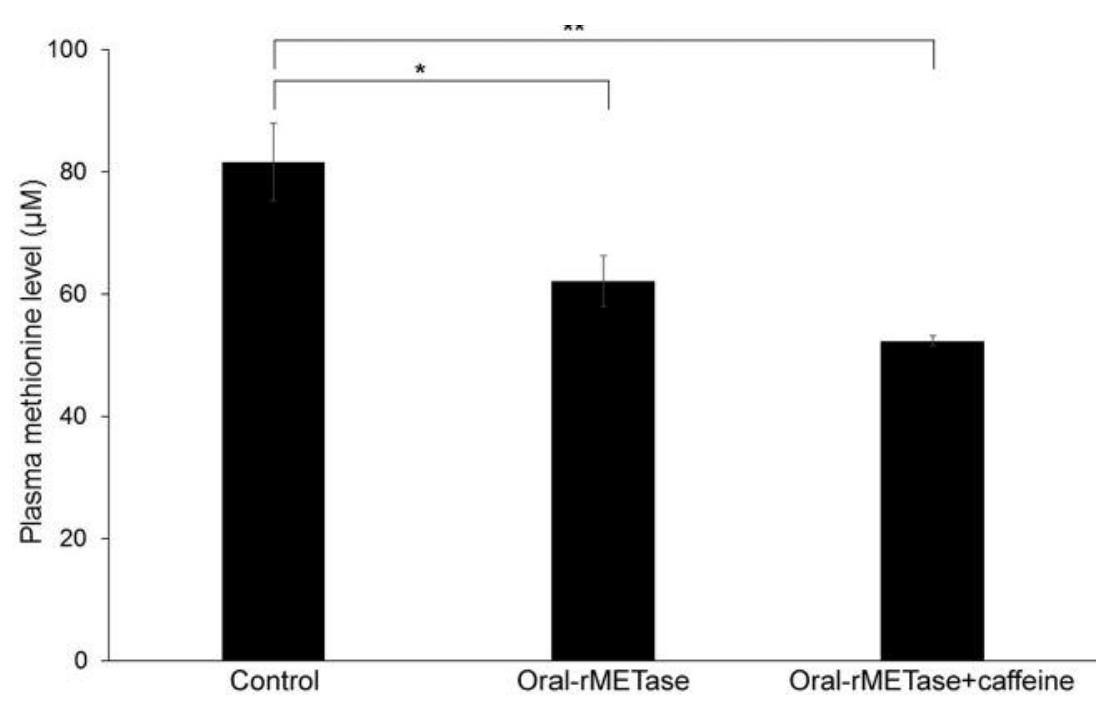

Figure 3. Plasma MET levels at the end of treatment of mice with synovial sarcoma PDOX with o-rMETase alone, and o-rMETase combined with caffeine, compared to the control group. $n=3$ mice/group. Significantly different at $* p<0.05$, and $* * p<0.01$. Error bars: $\pm S E M$.

Pre-treatment

Dost-treatment

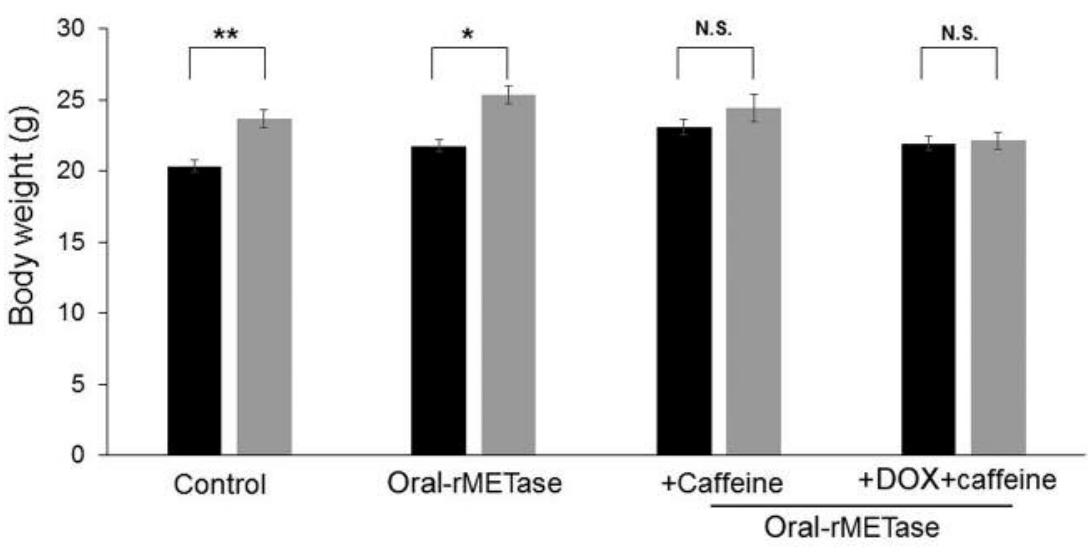

Figure 4. Body weight of mice bearing synovial sarcoma PDOX. Bar graphs show mouse body weight for each treatment or control group pre-and post-treatment. Significantly different at $* p<0.05$, and $* * p<0.01$. N.S., not significant. Error bars: \pm SEM.

SS-PDOX. The SS-PDOX was previously shown to be DOX resistant (7). Figures 1 and 2 show that all treatments significantly inhibited SS-PDOX compared to the untreated control (rMETase alone: $p=0.004$, o-rMETase combined with caffeine: $p<0.001$, DOX combined with caffeine and rMETase: $p<0.001$ ) on day 14 after initiation of treatment. Tumor regression was observed only in the mice treated with DOX combined with caffeine and o-rMETase (Figures 1 and 2).

Effect of rMETase on plasma methionine level. Plasma Lmethionine levels were significantly reduced after o-rMETase treatment $(p=0.048)$ and combination treatment of o-rMETase and caffeine $(p=0.008)$ (Figure 3$)$. The combination of orMETase and caffeine tended to reduce the MET level more than o-rMETase alone $(p=0.33)$ (Figure 3).

Effect of treatment on body weight. Mouse body weight was measured pre-treatment and post-treatment. There was no body weight loss and no significant difference in body weight pre-and post-treatment in any treatment group (Figure 4). There were no other observed side-effects or animal deaths in any group. 


\section{Discussion}

The present results suggest that this SS-PDOX is METdependent since it responded to o-rMETase. Combining caffeine with o-rMETase increased its efficacy against the SS-PDOX tumor, which was further strongly enhanced by adding DOX.

Methionine dependence of cancer is due to excess use of methionine for aberrant transmethylation reactions of cancer cells (16). This excess use of methionine by cancer cells is termed the Hoffman effect and may be analogous to the Warburg effect for excess use of glucose in cancer. Positronemission tomography imaging (PET) of cancer with $\left[{ }^{11} \mathrm{C}\right]-$ methionine gives a stronger signal than does $\left[{ }^{18} \mathrm{~F}\right]-$ fluorodeoxyglucose (17), indicating the Hoffman effect is stronger than the Warburg effect (18).

The combination treatment with o-rMETase and caffeine along with DOX was much more effective than DOX alone and induced regression of the SS-PDOX tumor, perhaps due to induction of mitotic catastrophe by caffeine on rMETase $\mathrm{S} / \mathrm{G}_{2}$-arrested cancer cells. This combination treatment of orMETase caffeine with DOX, a first-line drug for soft-tissue sarcoma treatment, overcame DOX-resistance in SS without observable toxicity.

Wang et al. reported that caffeine enhances cisplatininduced lung cancer cell death by inhibiting expression of the ataxia telangiectasia and RAD3-related protein (ATR), which resulted in increased expression of the pro-apoptotic protein p53-up-regulated modulator of apoptosis (PUMA) (19). Caffeine potentiated chemotherapy for osteosarcoma $(12,20-22)$, high-grade soft-tissue sarcoma (23), metastatic carcinoma and lymphoma of the bone and soft tissue (24), and hepatocellular carcinoma cells (25). Caffeine together with adenovirus-mediated transfer of phosphatase and tensin homolog (Ad-PTEN) suppressed cell growth and induced apoptosis in colorectal cancer cells (26). Caffeine also inhibited acetaldehyde-induced hepatic stellate cell activation (27) and paclitaxel-induced apoptosis of colorectal cancer cells (28). Caffeine inhibited tumor growth and elevated pro-inflammatory cytokine production during tumor initiation, through the antagonism of the $\mathrm{A}_{2 \mathrm{~A}}$ receptor (29). Caffeine can be used as a DNA damagesensitizing agent in cancer cells (30) and enhances the removal of DNA-damaged cells (31). Cafeine was also shown to induce sustained apoptosis of gastric cancer cells and function as an anticancer agent by activating the caspase-9/-3 pathway (32). Caffeine together with idelalisib reduced the immunosuppressive activity of activated chronic lymphocytic leukemia cells by inhibiting phosphoinositide 3-kinase $\delta$ (33). In an orthotopic xenograft animal model for glioblastoma, Cheng et al. reported that caffeine reduced tumor size, proliferation, and invasion of glioma cells through the Rho-associated coiled-coil kinase (ROCK)-cathepsin B/focal adhesion kinase (FAK)/extracellular-signal-regulated kinase (ERK) signaling pathway (34).

In conclusion, we determined o-rMETase inhibited tumor growth of an SS-PDOX, and that combining rMETase with caffeine increased its efficacy. Adding DOX to this combination led to regression of the SS-PDOX tumor and overcame DOX resistance of the SS suggesting important clinical potential of this strategy.

\section{Conflicts of Interest}

The Authors declare that there are no potential conflicts of interest in regard to this study.

\section{References}

1 Rajwanshi A, Srinivas R and Upasana G: Malignant small round cell tumors. J Cytol 26: 1-10, 2009.

2 Sultan I, Rodriguez-Galindo C and Saab R, Yasir S, Casanova $\mathrm{M}$ and Ferrari A. Comparing children and adults with synovial sarcoma in the Surveillance, Epidemiology, and End Results program, 1983 to 2005: an analysis of 1268 patients. Cancer 115: 3537-3547, 2009.

3 Vining CC, Sinnamon AJ, Ecker BL, Kelz RR, Fraker DL, Roses RE and Karakousis GC: Adjuvant chemotherapy in resectable synovial sarcoma. J Surg Oncol 116: 550-558, 2017.

4 Hoffman RM. Patient-derived orthotopic xenografts: better mimic of metastasis than subcutaneous xenografts. Nat Rev Cancer 15: 451-452, 2015.

5 Igarashi K, Kawaguchi K, Kiyuna T, Miyake K, Miyake M, Li S, Han Q, Tan Y, Zhao M, Li Y, Nelson SD, Dry SM, Singh AS, Elliott IA, Russell TA, Eckardt MA, Yamamoto N, Hayashi K, Kimura H, Miwa S, Tsuchiya H, Eilber FC and Hoffman RM: Tumor-targeting Salmonella typhimurium A1-R combined with recombinant methioninase and cisplatinum eradicates an osteosarcoma cisplatinum-resistant lung metastasis in a patientderived orthotopic xenograft (PDOX) mouse model: decoy, trap and kill chemotherapy moves toward the clinic. Cell Cycle 17: 801-809, 2018.

6 Igarashi K, Kawaguchi K, Li S, Han Q, Tan Y, Murakami T, Kiyuna T, Miyake K, Miyake M, Singh AS, Eckardt MA, Nelson SD, Russell TA, Dry SM, Li Y, Yamamoto N, Hayashi K, Kimura H, Miwa S, Tsuchiya H, Singh SR, Eilber FC and Hoffman RM: Recombinant methioninase in combination with doxorubicin overcomes first-line doxorubicin resistance in a patient-derived orthotopic xenograft nude-mouse model of undifferentiated spindle-cell sarcoma. Cancer Lett 417: 168$173,2018$.

7 Igarashi K, Kawaguchi K, Li S, Han Q, Tan Y, Gainor E, Kiyuna T, Miyake K, Miyake M, Higuchi T, Oshiro H, Singh AS, Eckardt MA, Nelson SD, Russell TA, Dry SM, Li Y, Yamamoto N, Hayashi K, Kimura H, Miwa S, Tsuchiya H, Eilber FC and Hoffman RM: Recombinant methioninase combined with doxorubicin (doxorubicin) regresses a doxorubicin-resistant synovial sarcoma in a patient-derived orthotopic xenograft (PDOX) mouse model. Oncotarget 9: 19263-19272, 2018. 
8 Igarashi K, Li S, Han Q, Tan Y, Kawaguchi K, Murakami T, Kiyuna T, Miyake K, Li Y, Nelson SD, Dry SM, Singh AS, Elliott IA, Russell TA, Eckardt MA, Yamamoto N, Hayashi K, Kimura H, Miwa S, Tsuchiya H, Eilber FC and Hoffman RM: Growth of a doxorubicin-resistant undifferentiated spindle-cell sarcoma PDOX is arrested by metabolic targeting with recombinant methioninase. J Cell Biochem 119: 3537-3544, 2018.

9 Hoffman RM. Development of recombinant methioninase to target the general cancer-specific metabolic defect of methionine dependence: A 40-year odyssey. Expert Opin Biol Ther 15: 2131, 2015.

10 Kawaguchi K, Miyake K, Han Q, Li S, Tan Y, Igarashi K, Kiyuna T, Miyake M, Higuchi T, Oshiro H, Zhang Z, Razmjooei $S$, Wangsiricharoen S, Bouvet $M$, Singh SR, Unno $M$ and Hoffman RM: Oral recombinant methioninase (rMETase) is superior to injectable rMETase and overcomes acquired gemcitabine resistance in pancreatic cancer. Cancer Lett 432: 251-259, 2018.

11 Miwa S, Yano S, Tome Y, Sugimoto N, Hiroshima Y, Uehara F, Mii S, Kimura H, Hayashi K, Efimova EV, Fujiwara T, Tsuchiya $\mathrm{H}$ and Hoffman RM: Dynamic color-coded fluorescence imaging of the cell-cycle phase, mitosis, and apoptosis demonstrates how caffeine modulates cisplatinum efficacy. J Cell Biochem 114: 2454-2460, 2013

12 Igarashi K, Kawaguchi K, Kiyuna T, Murakami T, Miwa S, Nelson SD, Dry SM, Li Y, Singh AS, Kimura H, Hayashi K, Yamamoto N, Tsuchiya H, Eilber FC and Hoffman RM: Efficacy in vitro of caffeine and valproic acid on patient-derived undifferentiated pleomorphic sarcoma and rhabdomyosarcoma cell lines. Anticancer Res 37: 4081-4084, 2017.

13 Ehrhardt H, Wachter F, Grunert M and Jeremias I: Cell cyclearrested tumor cells exhibit increased sensitivity towards TRAIL-induced apoptosis. Cell Death Dis 4: e661, 2013.

14 Kawaguchi K, Igarashi K, Li S, Han Q, Tan Y, Miyake K, Kiyuna T, Miyake M, Murakami T, Chmielowski B, Nelson SD, Russell TA, Dry SM, Li Y, Unno M, Eilber FC and Hoffman RM: Recombinant methioninase (rMETase) is an effective therapeutic for $B R A F$-V600E-negative as well as-positive melanoma in patient-derived orthotopic xenograft (PDOX) mouse models. Oncotarget 9: 915-923, 2017.

15 Tan Y, Xu M, Tan X, Tan X, Wang X, Saikawa Y, Nagahama T, Sun X, Lenz M and Hoffman RM: Overexpression and largescale production of recombinant L-methionine-alpha-deaminogamma-mercaptomethane-lyase for novel anticancer therapy. Protein Expr Purif 9: 233-245, 1997.

16 Stern PH and Hoffman RM: Elevated overall rates of transmethylation in cell lines from diverse human tumors. In Vitro 20: 663-670, 1984.

$17 \mathrm{Xu} \mathrm{W}$, Gao L, Shao A, Zheng J and Zhang J: The performance of ${ }^{11} \mathrm{C}$-MET PET in the differential diagnosis of glioma recurrence. Oncotarget 8: 91030-91039, 2017.

18 Hoffman RM: Patient-derived orthotopic xenografts: better mimic of metastasis than subcutaneous xenografts. Nat Rev Cancer 15: 451-452, 2015

19 Wang G, Bhoopalan V, Wang D, Wang L and Xu X: The effect of caffeine on cisplatin-induced apoptosis of lung cancer cells. Exp Hematol Oncol 4: 5, 2015.

20 Tsuchiya H, Yasutake H, Yokogawa A, Baba H, Ueda Y and Tomita K: Effect of chemotherapy combined with caffeine for osteosarcoma. J Cancer Res Clin Oncol 118: 567-569, 1992.
21 Igarashi K, Yamamoto N, Hayashi K, Takeuchi A, Kimura H, Miwa S, Hoffman RM and Tsuchiya H: Non-toxic efficacy of the combination of caffeine and valproic acid on human osteosarcoma cells in vitro and in orthotopic nude-mouse models. Anticancer Res 36: 4477-4482, 2016.

22 Igarashi K, Kawaguchi K, Kiyuna T, Murakami T, Yamamoto N, Hayashi K, Kimura H, Miwa S, Tsuchiya H and Hoffman RM: Antimetastatic efficacy of the combination of caffeine and valproic acid on an orthotopic human osteosarcoma cell line model in nude mice. Anticancer Res 37: 1005-1011, 2017.

23 Takeuchi A, Tsuchiya H, Yamamoto N, Hayashi K, Yamauchi K, Kawahara M, Miyamoto K and Tomita K: Caffeine-potentiated chemotherapy for patients with high-grade soft tissue sarcoma: long-term clinical outcome. Anticancer Res 7: 3489-3495, 2007.

24 Hayashi M, Tsuchiya H, Yamamoto N, Karita M, Shirai T, Nishida H, Takeuchi A and Tomita K: Caffeine-potentiated chemotherapy for metastatic carcinoma and lymphoma of bone and soft tissue. Anticancer Res 25: 2399-2405, 2005.

25 Oda Y, Hidaka M and Suzuki A: Caffeine Has a Synergistic Anticancer Effect with Cisplatin via inhibiting Fanconi anemia group d2 protein monoubiquitination in hepatocellular carcinoma cells. Biol Pharm Bull 40: 2005-2009, 2017.

26 Saito Y, Gopalan B, Mhashilkar AM, Roth JA, Chada S, Zumstein $\mathrm{L}$ and Ramesh R: Adenovirus-mediated PTEN treatment combined with caffeine produces a synergistic therapeutic effect in colorectal cancer cells. Cancer Gene Ther 10: 803-813, 2003.

27 Wang H, Guan W, Yang W, Wang Q, Zhao H, Yang F, Lv X and Li J: Caffeine inhibits the activation of hepatic stellate cells induced by acetaldehyde via adenosine A2A receptor mediated by the cAMP/PKA/SRC/ERK1/2/P38 MAPK signal pathway. PLoS One 9: e92482, 2014.

28 Mhaidat NM, Alzoubi KH, Al-Azzam SI and Alsaad AA: Caffeine inhibits paclitaxel-induced apoptosis in colorectal cancer cells through the up-regulation of MCL-1 levels. Mol Med Rep 9: 243-248, 2014.

29 Eini H, Frishman V, Yulzari R, Kachko L, Lewis EC, Chaimovitz $\mathrm{C}$ and Douvdevani A: Caffeine promotes anti-tumor immune response during tumor initiation: Involvement of the adenosine A2A receptor. Biochem Pharmacol 98: 110-118, 2015.

30 Tsabar M, Eapen VV, Mason JM, Memisoglu G, Waterman DP, Long MJ, Bishop DK and Haber JE: Caffeine impairs resection during DNA break repair by reducing the levels of nucleases SAE2 and DNA2. Nucleic Acids Res 43: 6889-901, 2015.

$31 \mathrm{Lu}$ YP, Lou YR, Peng QY, Xie JG, Nghiem P and Conney AH: Effect of caffeine on the ATR/CHK1 pathway in the epidermis of UVB-irradiated mice. Cancer Res 68: 2523-2529, 2008.

32 Liu H, Zhou Y and Tang L: Caffeine induces sustained apoptosis of human gastric cancer cells by activating the caspase-9/caspase3 signalling pathway. Mol Med Rep 16: 2445-2454, 2017.

33 Hock BD, MacPherson SA and McKenzie JL: Idelalisib and caffeine reduce suppression of T-cell responses mediated by activated chronic lymphocytic leukemia cells. PLoS One 12: e0172858, 2017.

34 Cheng YC, Ding YM, Hueng DY, Chen JY and Chen Y: Caffeine suppresses the progression of human glioblastoma via cathepsin B and MAPK signaling pathway. J Nutr Biochem 33: 63-72, 2016.

Received September 7, 2018

Revised September 18, 2018

Accepted September 21, 2018 